\title{
Extensive lymphadenopathy leading to cancerous pericardial effusion and tamponade in a T1 pancreatic head adenocarcinoma: a case report
}

\author{
Mohamed Abdelgawad ${ }^{1}$, Mahmoud Omar $^{2} \wedge$, Hadeer Eltahan $^{3}$, Tyler Davis ${ }^{1}$, Lutfi Barghuthi $^{1}$, \\ Emad Kandil' ${ }^{2}$, Hisham Ismael ${ }^{1}$ \\ ${ }^{1}$ Department of Surgery, University of Texas Health Science Center, UT Health East Texas, Tyler, TX, USA; ${ }^{2}$ Department of Surgery, School of \\ Medicine, Tulane University, New Orleans, LA, USA; ${ }^{3}$ Montefiore Medical Center, Albert Einstein College of Medicine, New York City, NY, USA \\ Correspondence to: Hisham Ismael, MD, FACS. Associate Professor of General and Hepatobiliary Surgery, Department of Surgery, University of Texas \\ Health Science Center, UT Health East Texas, Tyler, TX 75708, USA. Email: Hishaam.Ismael@uthct.edu.
}

\begin{abstract}
In 2019, Pancreatic cancer became the third leading cause of cancer related mortality in the United States of America. Pancreatic cancer is a fatal malignancy that is predominantly seen in men with advanced age with aggressive course. Ninety percent of cases are adenocarcinoma. Pancreatic adenocarcinoma is very difficult to detect as is usually asymptomatic with no early signs. At least $80 \%$ of tumors are unresectable by the time of diagnosis. Despite substantial improvement in the survival rates for other major cancer forms, pancreatic cancer survival rates have remained relatively unchanged since 1960s. Multidisciplinary therapy with a combination of chemotherapy, radiation and surgery performed at high volume centers remains the best chance for cure. We report a rare case of a small T1 pancreatic head carcinoma with underlying chronic pancreatitis presenting with cancerous pericardial effusion and tamponade. Despite the small size of the primary tumor, the extensive pattern of lymphatic occlusion can lead to mesenteric and mediastinal lymphatic spread to the pericardium. US guided emergent pericardiocentesis was performed with removal of $750 \mathrm{cc}$ of serosanguinous fluid. Cytopathological examination of the fluid revealed poorly differentiated pancreatic adenocarcinoma. A Port-A-Cath was placed, and he was referred to the oncology department to start chemotherapy.
\end{abstract}

Koywords: Pancreatic adenocarcinoma; pericardial metastasis; cardiac tamponade; lymphadenopathy; case report

Submitted Sep 28, 2021. Accepted for publication Dec 15, 2021.

doi: $10.21037 / g s-21-670$

View this article at: https://dx.doi.org/10.21037/gs-21-670

\section{Introduction}

Adenocarcinoma is the most common malignant pancreatic tumor, affecting the head of the pancreas in $60-70 \%$ of cases (1). Pancreatic adenocarcinoma currently is the $3 \mathrm{rd}$ leading cause of cancer related mortality in the USA (2). By the time of diagnosis, at least $80 \%$ of tumors are unresectable (3). The etiology of pancreatic cancer is complex and multifactorial. In addition to cigarette smoking and family history, chronic pancreatitis is a well-known non modifiable risk factor (4). Pancreatic cancer is an extremely challenging disease with surgery offering the only chance for cure.

Surgery followed by adjuvant chemotherapy is the standard of care for resectable pancreatic cancer (5). Preoperative axial imaging with high resolution computed tomography (CT), magnetic resonance imaging (MRI), and endoscopic ultrasonography (EUS) are essential for tumor staging, and assessment of resectability. Tumors that encase the superior mesenteric artery (SMA) or the presence of extensive lymphadenopathy outside the field

^ ORCID: Mahmoud Omar, 0000-0001-7917-7973; Lutfi Barghuthi, 0000-0002-3712-4280. 


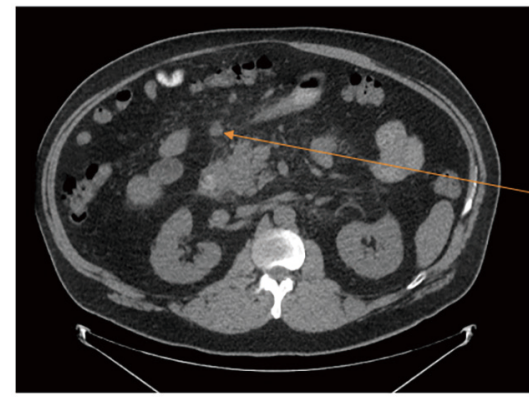

Non-specific lymphadenopathy attributed to pancreatitis

Figure 1 CT abdomen demonstrating pancreatic lesion, with nonspecific lymphadenopathy. CT, computed tomography.

of resection are signs of locally advanced and unresectable disease (3). Lymph nodes greater than $1 \mathrm{~cm}$ in diameter are generally considered metastatic especially in the setting of large tumors (6). Most small tumors with no evidence of lymphadenopathy can be treated surgically with a Whipple pancreaticoduodenectomy (5). Kanda et al. (7) reported a higher chance of distant lymph node metastasis with larger primary tumors (T3, T4). However, small T1 or T2 tumors had only regional lymph nodes metastasis.

Hereby, we report a case of a small T1 pancreatic head adenocarcinoma with no clear evidence of metastatic lymphadenopathy on preoperative CT or EUS. Intraoperatively, extensive mesenteric lymphadenopathy outside the field of surgery and lymphatic occlusion was identified causing the resection to be aborted. Within a month, the patient presented with cancerous pericardial effusion leading to tamponade. We present the following case in accordance with the CARE reporting checklist (available at https:// gs.amegroups.com/article/view/10.21037/gs-21-670/rc).

\section{Case presentation}

A 48-year-old male with a history of diabetes, hypertension, and chronic pancreatitis presented to our hospital with upper abdominal pain radiating to his back with greasy meals and $60 \mathrm{lbs}$. weight loss over a 3 -month period. CT demonstrated a hypo-attenuating lesion in the pancreatic head measuring $1.6 \mathrm{~cm} \times 1.5 \mathrm{~cm}$, and nonspecific mesenteric lymphadenopathy (Figure 1). Triphasic abdominal CT noted mild peripancreatic edema, with hypo-enhancing abnormal pancreatic head lesion and small peripancreatic lymph nodes, with no enhancing liver lesion, no biliary dilation. EUS did not show suspicious lymph nodes and the biopsy of the primary pancreatic lesion demonstrated adenocarcinoma. At time of diagnosis, the CA 19-9 was 394 units $/ \mathrm{mL}$, alpha-fetoprotein was $3.4 \mathrm{ng} / \mathrm{mL}$.

The enlarged lymph nodes at the root of the mesentery were considered reactive secondary to pancreatitis especially that the primary tumor was small and there were no biliary or pancreas ductal dilation. The case was discussed at our Multidisciplinary Tumor Board, and the plan was to proceed with a Whipple procedure.

A diagnostic laparoscopy ruled out carcinomatosis, then converted to laparotomy to assess resectability. We identified a lesion in the left lateral section of the liver that was biopsied and revealed a benign bile duct hamartoma. During the dissection, we identified bulky lymph nodes at the neck of the pancreas surrounding the superior mesenteric vein (SMV) and SMA. The lymphadenopathy extended across the root of the mesentery to the inferior mesenteric vein (IMV) at the ligament of Treitz. The jejunum appeared milky with prominent chylous channels suggesting lymphatic obstruction. The lymphadenopathy involved the ileocolic pedicle as well the inferior mesenteric artery (IMA) distribution towards the sigmoid colon. The common hepatic artery station 8 lymph node was resected, and pathology reported metastatic poorly differentiated adenocarcinoma. Therefore, the Whipple was aborted. Prophylactic gastro-jejunostomy bypass was done for anticipated gastric outlet obstruction.

Two weeks later, the patient returned to the emergency department with worsening nausea, vomiting, and shortness of breath. He was tachycardic and had muffled heart sounds. The following differential diagnoses were considered; pericardial effusion with developing tamponade, or pulmonary embolism given the history of pancreatic tumor. CT chest with contrast noted a large pericardial effusion (Figure 2). Shortly after, he developed hypotension and a diagnosis of pericardial tamponade was established. Therefore, an US guided emergent pericardiocentesis was performed with removal of $750 \mathrm{cc}$ of serosanguinous fluid. Cytopathological examination of the fluid revealed poorly differentiated pancreatic adenocarcinoma (Figure 3). A Port-A-Cath was placed, and he was started on chemotherapy.

All procedures detailed in this case report were in accordance with the ethical standards of the institutional and/or national research committee(s) and with the Helsinki Declaration (as revised in 2013). Written informed consent was obtained from the patient for publication of this case report and accompanying images. A copy of the written consent is available for review by the 
editorial office of this journal.

\section{Discussion}

Pancreatic adenocarcinoma is an aggressive malignancy with a high propensity for metastasis (3). Surgery combined with chemotherapy, and radiation are the only potentially curative treatment but must be integrated into a complete multidisciplinary treatment sequence to optimize its effectiveness (5).

The peripancreatic regional lymph nodes are frequently involved by disease (8). Larger tumor size (T stage) is widely acknowledged to be associated with increased lymph node metastasis $(7,9)$. Resectable pancreatic cancers are typically in the earliest stages of the disease (stage $1 \mathrm{~A}, 1 \mathrm{~B}$ or $2 \mathrm{~A}$ ) and any nodal involvement outside the field of disease indicates unresectable disease (6).

Preoperative CT for staging of pancreatic cancer

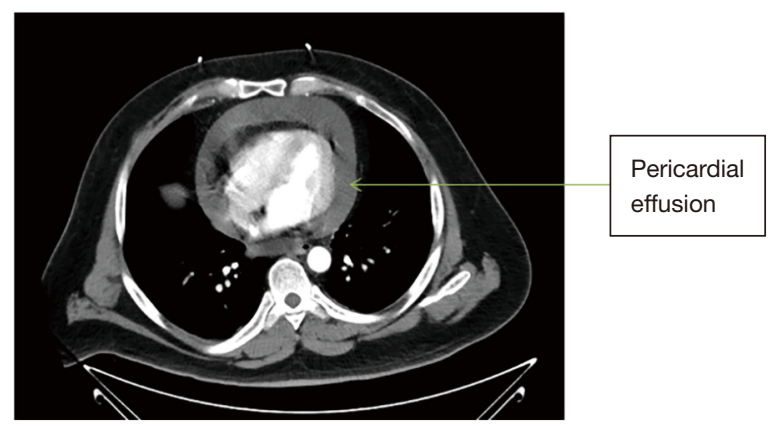

Figure 2 CT chest with contrast indicating a pericardial effusion. CT, computed tomography. identifies metastatic lymph nodes based on the nodal size $>1 \mathrm{~cm}$ (6). However, Prenzel et al. (10) reported that nodal maximal diameter does not correlate with metastatic potential demonstrating that $67 \%$ of cytopathologically proven metastatic nodes measured less than $5 \mathrm{~mm}$.

Our patient's preoperative CT scan noted enlarged lymph nodes. However, given the patients age and functional status, the small size of the primary lesion (T1) and his history of pancreatitis, these were considered likely reactive. We elected to proceed with curative resection to improve his chances of survival. Prenzel et al. (10) reported that $70 \%$ of the larger size lymph node were cytopathologically tumor free.

Lymph node metastasis may lead to lymphatic obstruction and impaired drainage. We believe the patient's abdominal pain with fatty meals was secondary to lymphatic obstruction rather than biliary/pancreatic ductal occlusion or perineural involvement as supported by preoperative imaging.

Tumors that are most likely to involve the heart and pericardium include cancers of the lung and breast, melanoma, and lymphoma. Pericardial effusion secondary to metastatic spread can be facilitated by different mechanisms: elevated hydrostatic pressure secondary to lymphatic and venous obstruction, the detrimental impact of cachexia and subsequent hypoalbuminemia leading to low oncotic pressure, or pericardial irritation and over production of exudative fluid secondary to tumor cells implantation potentially leading to tamponade (11).

Multiple studies reported metastatic pericardial effusion secondary to primary gastrointestinal tumors through direct, hematological, and lymphatic spread. Retrograde

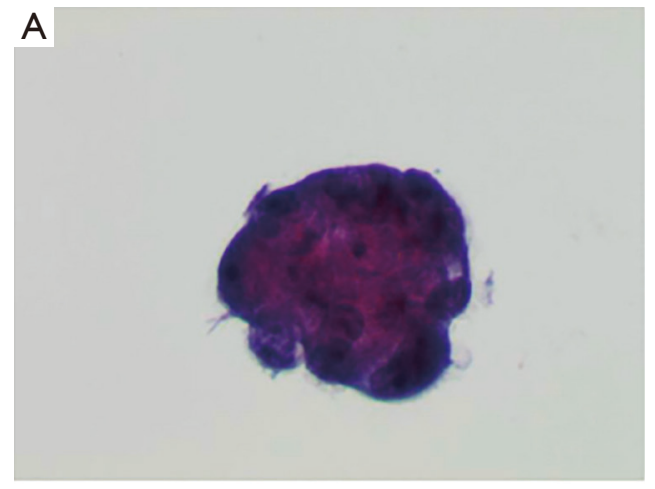

Cluster of malignant cells on Thinprep slide $(H \& E, \times 400)$

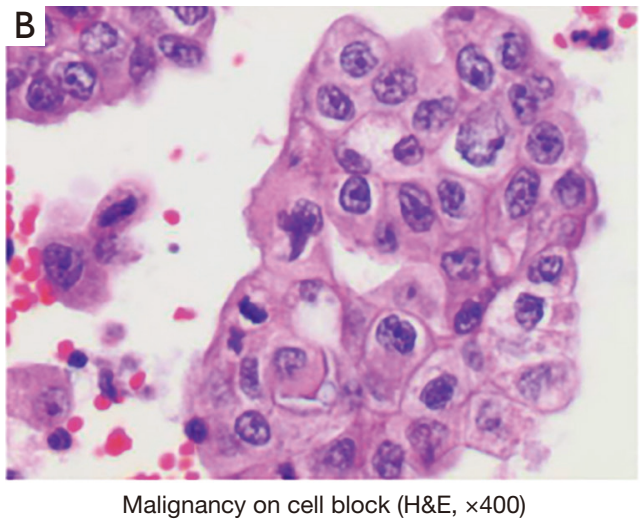
Figure 3 Pericardial fluids with pancreatic adenocarcinoma on H\&E. (A) Cluster of malignant cells on ThinPrep slide; (B) malignancy on cell block. Staining method: H\&E. Magnification: $\times 400$. 
lymphatic spread to the pericardium secondary to colon and gastric primary tumors has been previously reported (11-13). Kiryu et al. (14) reported the first case of pancreatic head cancer presenting with cancerous pericardial effusion and tamponade. Their report indicated lymphatic and vascular invasion on autopsy. However, their patient had a large tumor with multiple organ metastases. Our patient has a $1.6-\mathrm{cm}$ tumor with significant mesenteric adenopathy and cancerous pericardial effusion. These findings suggest lymphatic spread to the pericardium.

Bergquist et al. (15) reported that elevated CA 19-9 is associated with decreased stage-specific survival compared to non-secretors and those with normal CA 19-9 levels. The authors also reported that neoadjuvant therapy then curative intent surgery best mitigates the increased mortality hazard. However, the NCC guidelines version 2.2021 for pancreatic adenocarcinoma treatment as well as American Society of Clinical Oncology Clinical Practice Guideline Update stated that the standard treatment for resectable pancreatic adenocarcinoma currently remains upfront surgery followed by adjuvant chemotherapy $(16,17)$. Furthermore, no clinical trials suggested such findings, and the guidelines are yet to endorse these recommendations.

The NCC guidelines also recommended laparotomy $v s$. minimally invasive procedure for surgical management. We started with laparoscopy to rule out carcinomatosis, then proceeded with laparotomy to assess resectability. When deemed unresectable, we did the gastric bypass to avoid gastric outlet obstruction. Croome et al. (18) reported that total laparoscopic pancreaticoduodenectomy is associated with shorter hospital stay, shorter interval to start postoperative chemotherapy. However, our patient recovered in a timely manner. Furthermore, the patient developed the cancerous pericardial effusion only 2 weeks following the operation. We believe that having minimally invasive approach could not have altered the patient outcomes in this case.

\section{Conclusions}

Our case report demonstrates that pancreatic cancer, even when the primary tumor is small, can present with significant lymphadenopathy, lymphatic obstruction and spread to the pericardium with subsequent cancerous pericardial effusion. In a patient with early pancreatic head adenocarcinomas, mesenteric lymph node metastasis represents an uncommon, but clinically significant finding that can lead to drastic changes in the treatment plan, and outcomes.

\section{Acknowledgments}

Funding: None.

\section{Footnote}

Reporting Checklist: The authors have completed the CARE reporting checklist. Available at https://gs.amegroups.com/ article/view/10.21037/gs-21-670/rc

Peer Review File: Available at https://gs.amegroups.com/ article/view/10.21037/gs-21-670/prf

Conflicts of Interest: All authors have completed the ICMJE uniform disclosure form (available at https://gs.amegroups. com/article/view/10.21037/gs-21-670/coif). EK serves as an Editor-in-Chief of Gland Surgery. The other authors have no conflicts of interest to declare.

Ethical Statement: The authors are accountable for all aspects of the work in ensuring that questions related to the accuracy or integrity of any part of the work are appropriately investigated and resolved. All procedures performed in studies involving human participants were in accordance with the ethical standards of the institutional and/or national research committee(s) and with the Helsinki Declaration (as revised in 2013). Written informed consent was obtained from the patient for publication of this case report and accompanying images. A copy of the written consent is available for review by the editorial office of this journal.

Open Access Statement: This is an Open Access article distributed in accordance with the Creative Commons Attribution-NonCommercial-NoDerivs 4.0 International License (CC BY-NC-ND 4.0), which permits the noncommercial replication and distribution of the article with the strict proviso that no changes or edits are made and the original work is properly cited (including links to both the formal publication through the relevant DOI and the license). See: https://creativecommons.org/licenses/by-nc-nd/4.0/.

\section{References}

1. Winer LK, Dhar VK, Wima K, et al. The Impact of Tumor Location on Resection and Survival for Pancreatic 
Ductal Adenocarcinoma. J Surg Res 2019;239:60-6.

2. Ferlay J, Ervik M, Lam F, et al. Global Cancer Observatory: Cancer Today. Lyon: International Agency for Research on Cancer. Available online: https://gco.iarc. fr/today

3. Vincent A, Herman J, Schulick R, et al. Pancreatic cancer. Lancet 2011;378:607-20.

4. Midha S, Chawla S, Garg PK. Modifiable and nonmodifiable risk factors for pancreatic cancer: A review. Cancer Lett 2016;381:269-77.

5. Martin-Perez E, Domínguez-Muñoz JE, Botella-Romero F, et al. Multidisciplinary consensus statement on the clinical management of patients with pancreatic cancer. Clin Transl Oncol 2020;22:1963-75.

6. Midwinter MJ, Beveridge CJ, Wilsdon JB, et al. Correlation between spiral computed tomography, endoscopic ultrasonography and findings at operation in pancreatic and ampullary tumours. Br J Surg 1999;86:189-93.

7. Kanda M, Fujii T, Nagai S, et al. Pattern of lymph node metastasis spread in pancreatic cancer. Pancreas 2011;40:951-5.

8. Fujita T, Nakagohri T, Gotohda N, et al. Evaluation of the prognostic factors and significance of lymph node status in invasive ductal carcinoma of the body or tail of the pancreas. Pancreas 2010;39:e48-54.

9. Pu N, Chen Q, Gan W, et al. Lymph Node Metastatic Patterns and Survival Predictors Based on Tumor Size in Pancreatic Ductal Adenocarcinoma. Adv Ther 2021;38:4258-70.

10. Prenzel KL, Hölscher AH, Vallböhmer D, et al. Lymph node size and metastatic infiltration in adenocarcinoma of the pancreatic head. Eur J Surg Oncol 2010;36:993-6.

11. Scheinin SA, Sosa-Herrera J. Case report: cardiac

Cite this article as: Abdelgawad M, Omar M, Eltahan H, Davis T, Barghuthi L, Kandil E, Ismael H. Extensive lymphadenopathy leading to cancerous pericardial effusion and tamponade in a $\mathrm{T} 1$ pancreatic head adenocarcinoma: a case report. Gland Surg 2022;11(1):285-289. doi: 10.21037/gs-21-670 tamponade resembling an acute myocardial infarction as the initial manifestation of metastatic pericardial adenocarcinoma. Methodist Debakey Cardiovasc J 2014;10:124-8.

12. Pãosinho A, Esteves AL, Pereira AJ. From the Gut to the Heart: Cardiac Tamponade due to Lymphatic Metastasis. Eur J Case Rep Intern Med 2019;6:001033.

13. Chen YG, Lai MJ, Lin YJ, et al. Complicating pericarditis and cardiac tamponade in a patient of primary HER2/ neu positive gastric signet ring cell carcinoma. Journal of Cancer Research and Practice 2017;4:59-62.

14. Kiryu $S$, Ito Z, Ishikawa $M$, et al. Cancerous pericarditis presenting as cardiac tamponade in a 68-year-old man with pancreatic adenocarcinoma: a case report. J Med Case Rep 2020;14:213.

15. Bergquist JR, Puig CA, Shubert CR, et al. Carbohydrate Antigen 19-9 Elevation in Anatomically Resectable, Early Stage Pancreatic Cancer Is Independently Associated with Decreased Overall Survival and an Indication for Neoadjuvant Therapy: A National Cancer Database Study. J Am Coll Surg 2016;223:52-65.

16. Tempero MA, Malafa MP, Al-Hawary M, et al. Pancreatic Adenocarcinoma, Version 2.2021, NCCN Clinical Practice Guidelines in Oncology. J Natl Compr Canc Netw 2021;19:439-57.

17. Khorana AA, Mangu PB, Berlin J, et al. Potentially Curable Pancreatic Cancer: American Society of Clinical Oncology Clinical Practice Guideline Update. J Clin Oncol 2017;35:2324-8.

18. Croome KP, Farnell MB, Que FG, et al. Total laparoscopic pancreaticoduodenectomy for pancreatic ductal adenocarcinoma: oncologic advantages over open approaches? Ann Surg 2014;260:633-8; discussion 638-40. 